\title{
Agricultural Model for Allocation of Crops Using Pollination Intelligence Method
}

\author{
C. N. Ejieji and A. E. Akinsunmade $(D)$ \\ Department of Mathematics, Faculty of Physical Sciences, University of Ilorin, Ilorin, Nigeria \\ Correspondence should be addressed to A. E. Akinsunmade; akintayoakinsunmade@gmail.com
}

Received 19 August 2019; Revised 9 May 2020; Accepted 18 May 2020; Published 1 June 2020

Academic Editor: Christian W. Dawson

Copyright (C) 2020 C. N. Ejieji and A. E. Akinsunmade. This is an open access article distributed under the Creative Commons Attribution License, which permits unrestricted use, distribution, and reproduction in any medium, provided the original work is properly cited.

\begin{abstract}
An agricultural model for allocation of crops is considered in this work using Pollination Intelligence Method. The model was constructed to solve farmer's decision making in allocating crops to a piece of land using market price, known yield of crops, cost incurred during planting, and the total amount of land available. A new class of metaheuristic method called Flower Pollinated Algorithm is also presented in this work to solve the designed model. An improved version of the Flower Pollinated Algorithm called Pollination Intelligence Algorithm using an iterative scheme to override the switch parameter in Flower Pollinated Algorithm is also presented and used in solving the designed model. A case study of a farmer in Ife, Osun State, Nigeria, was used to implement the model, and the results obtained suggested that instead of allocating crops to land randomly based on farmer's intuition, cost of planting, yield of crops, and market price were factors that must be considered by farmers for optimal profit before planting crops.
\end{abstract}

\section{Introduction}

The roles of agriculture cannot be swept off in human life as agricultural practices have helped in provision of jobs, availability of raw materials for industrial uses, income generation for agrarian countries, to mention but a few. Some countries like Nigeria who have in time past diverted the nation's economy from agriculture after the discovery of oil in 1956 are now in recent years revisiting and paying much interest to agricultural practices as a result of the recent decline in the price of oil. For countries like Saudi Arabia, agricultural projects are being sponsored majorly via irrigation process to help improve cropping activities in the country and this has tremendously helped with increasing the nation's economy. It is worthwhile to note that agriculture is one of the factors for growing economy and this calls for proper attention. Land as a factor of production plays a vital role in agricultural practices, and farmers are always faced with the problem of decision making when it comes to choosing crops to be planted for a particular period and the amount of land needed for cultivation, intuition process although has been used in allocating crops to land, yet not optimally guaranteed. It is therefore still a big challenge for farmers to choose crops to be planted and the area of land to be assigned for cultivation. To achieve this, factors that enhance maximum profit such as crop yield, weather condition, price of crops influenced by market scale and demand, government policy on some crops, pest/diseases, and fertility of soil must be considered before planting crops on a piece of land, and this has to do with agricultural farm management as crop allocated to small cultivation space can eventually turn out to be the most productive, yielding huge profit amidst other selected crops planted by a farmer or group of farmers [1]. Farm management as carried out by farmers has been defined by Dillon [2] as the process by which resources and situations are manipulated by the farm manager in trying with less than full information, to achieve his or her goals. To tackle this problem, a number of optimization models have been developed by different authors, depending on their geographical zones. The first appearance of a model of such was done by Krishna [3] using known data from an Indian settlement, and this work has 
served as a basis and motivation for many research studies on land allocation and crop planning. Others include Keith [4] who suggested that the climate of a place is worth considering as a useful tool in maximizing techniques in farm planning, using linear programing approach. Campbell et al. [5] designed a cropland allocation model using linear programing technique and geographic information system. Sante and Crecente [6] and Wankhade and Lunge [7] also used linear programing approach as well as Ahmed et al. [8]. Meanwhile, most of these models were solved using traditional methods of optimization with few attention on the modern method of optimization. Modern methods of optimization are the most trending methods used in solving optimization problems and have been applied to virtually all fields of life including engineering, science, and finance to mention but a few. A significant number of these methods are algorithms that are derived from studying biological and environmental factors, human and animal activities, and chemical processes. Some of these methods include Genetic Algorithm (GA) developed by Holand [9]; Simulated Annealing (SA), developed by Kirkpatrick et al. [10]; Differential Evolution Method (DE) by Storn and Price [11]; and recently Firefly Algorithm (FA), an algorithm developed from studying the nature of fireflies and their light production by Yang [12]. Others include Wind Driven Optimization (WDO) by Bayraktar et al. [13], Brain Storm Optimization (BSO) by Shi [14], and Flower Pollinated Algorithm by Yang [15].

In recent years, models have been developed for solving problems relating to crop selection and land management, like the work of Sante and Crecente [6], Hassan et al. [16], Tanko et al. [17], Nedunchezhian and Thirunavukkarasu [18], and Manos et al. [19]. Moreover, linear programing technique has been highlighted by Igwe et al. [20], as an efficient method of resource allocation in production planning, particularly in achieving increased agricultural productivity, and this has been applied to determine optimum enterprise combination in the work of Bamiro et al. [21]. Researchers like Wankhade and Lunge [7] have likewise made use of linear programing techniques to determine optimum allocation of crops using Simplex Algorithm and Ahmed et al. [8] to optimize the cropping pattern in Saudi Arabia, Majeke et al. [22] to mention but a few. As said earlier, the modern method of optimization has not been fully explored in solving land allocation problems. Badarudin et al. [23] gave a valid conclusion that metaheuristic approaches are good methods for solving problems relating to agriculture and land allocation. Based on this conclusion, we seek further study on the work of the abovementioned authors. In this work, agricultural model was developed for optimal crop allocation using Pollination Intelligence Algorithm which was constructed by modifying an existing metaheuristic method and used to solve the crop allocation model.

\section{Agricultural Model for Crop Allocation}

We consider a farmer or group of farmers in a region having a large portion of land say 20 hectares which is suitable for planting different types of crops. Available land must be maximized in such a way that maximum profit is obtained from all crops planted, while considering market price, history of crop yield in the region, cost of planting, and harvesting, with a set target of meeting the demand of the region. The farmer's decision is to optimally allocate his crop of interest to a portion of land for cultivation.

$$
\operatorname{Maximize}\left[\sum_{i=1}^{I}\left(P_{i} Y_{i}-C_{i}\right) A_{i}\right]-F_{c}^{L},
$$

subject to

$$
\begin{gathered}
\sum_{i}^{I} A_{i} \leq L, \\
P_{i} A_{i} \geq Y_{i}^{p}, \\
A_{i} \geq 0 .
\end{gathered}
$$

From the above model, the objective function equation (1) maximizes the production of the farmer considering market price of crops, yield of crops, cost of planting the crops, and total cost of cultivating the land. The constraint equations (2)-(4) are designed to meet the land requirement in such a way that all crops' acreage must not be above the available land for cultivation, the crop acreage must be allocated in such a way that the farmer's production target is met, and crop acreage must be nonnegative. Before implementing the above model, the following assumptions are made:

(1) Available land was suitable for planting farmer's selected crop of interest

(2) Selected crops were known to be planted in farmer's region

(3) Cost for pesticides, herbicides, fertilizer, and irrigation was treated under variable cost throughout the period of planting

To solve this model, a new class of metaheuristic optimization method called Flower Pollinated Algorithm by Yang [15] was presented. Flower Pollinated Algorithm (FPA) as proposed by Yang [15] has the ability of solving different types of optimization problems, but has not been applied to solve land allocation models. The algorithm is based on the principle of pollination. The primary purpose of flower is ultimately reproduction. Pollen agents such as insects, birds, bats, and other animals tend to visit flowering plants having been attracted by its nature (bright colour and scent). Apart from this, abiotic factor can also be responsible for the transfer of pollen grain in flowering plants, and ten percent of the pollination process involves this process. There are over $\$ 2000$ \$ varieties of pollinators, and they tend to behave by moving randomly. Based on this characteristic of flower pollination, Yang [15] developed Flower Pollination Algorithm (FPA). For simplicity, the following rules were used:

(1) Biotic and cross-pollination can be considered as a process of global pollination process, and pollen- 
carrying pollinators move in a random direction which obeys Lévy flight

(2) Self-pollination and abiotic factors were used to generalize local pollination

(3) Pollinators such as insects can develop flower constancy which is equivalent to reproduction probability that is proportional to the similarity of two flowers involved

(4) The interaction of switching of local pollination and global pollination can be controlled by a switch probability $p \in[0,1]$, with a slight bias towards local pollination

From implementation point of view, a set of updating formulas are needed to convert the rules into updating equations. In global pollination, flower pollen gametes are carried by pollinators such as insects, and pollen can travel over a long distance because insects can often fly and move in a much longer range. Rule 1 and flower constancy can be represented as follows:

$$
x_{i}^{t+1}=x_{i}^{t}+L(\lambda)\left(g_{*}-x_{i}^{t}\right),
$$

where $x_{i}^{t}$ is the pollen or solution vector $x_{i}$ at iteration $t$ and $g_{*}$ is the current best solution found among all solutions at the current generation/iteration. $L(\lambda)$ is the parameter that corresponds to the strength of the pollinators which is essentially the step size. Since insects may move over a long distance, Lévy flight is designed to mimic this characteristic efficiently.

$L>0$ is drawn from a Lévy distribution:

$$
L \approx \frac{\lambda \Gamma(\lambda) \sin (\pi \lambda / 2)}{\pi} \frac{1}{s^{1+\lambda}}\left(s \geq s_{0}>0\right),
$$

where $\Gamma(\lambda)$ is the standard gamma function and this distribution is valid for large step $s>0$.

Rule 2 and Rule 3 can be represented as follows:

$$
x_{i}^{t+1}=x_{i}^{t}+e\left(x_{j}^{t}-x_{k}^{t}\right)
$$

where $x_{j}^{t}$ and $x_{k}^{t}$ are pollen from different flowers of the same plant species. This essentially mimics the flower constancy in a limited neighborhood. Mathematically, if $x_{j}^{t}$ and $x_{k}^{t}$ come from the flower type or selected from the same population, this equivalently becomes a local random walk if we draw $e$ from a uniform distribution in $[0,1]$. Though flower pollination activities can occur at all scales, both local and global, adjacent flower patches or flowers in the not so far away neighborhood are more likely to be pollinated by locally. In order to mimic this, we can effectively use a switch probability (Rule 4 ) or proximity probability $p$ to switch between common global pollination and intensive local pollination.

2.1. Pollination Intelligence Algorithm. Flower Pollination Algorithm (Algorithm 1) is limited to switching probabilities, thereby incapable of making a proper balance to search globally or locally. Experimentally, setting the probability $p=0.8$ works well for most application, and there is therefore need of increasing the exploration in such a way that the probability goes along with the random behavior of pollinating agents. To achieve this, an iterative scheme is defined to generate the value of $p$ at different stages of finding the best solution:

$$
p_{n+1}=\theta p_{n}\left(1-p_{n}\right),
$$

where $p \in(0,1)$ and $0<\theta \leq 4, n=(0,1,2, \ldots)$. The essence of introducing this scheme is to get rid of having a constant value of $p$ throughout the iteration compared to what was presented in Rule 4 of Flower Pollinated Algorithm.

Lévy flight has randomness in its step size which leads to huge variation in the position of the solution, at once it can have a long-range step and at another time it can have short steps. Due to this, Flower Pollinated Algorithm starts searching in a totally different search range and it may skip the global minima. To overcome this drawback and improve the exploration ability, Lévy flight is replaced by Sine Cosine operator. Sine Cosine Algorithm (SCA) as presented by Mirjalili [24] helps in searching promising areas of search space and we can deduce that pollinating agents tend to be distributed evenly in the search space:

$$
\begin{array}{ll}
x_{i}^{t+1}=x_{i}^{t}+r_{1} \sin \left(r_{2}\right)\left|r_{3} g_{*}-x_{i}^{t}\right|, & r_{4}<0.5, \\
x_{i}^{t+1}=x_{i}^{t}+r_{1} \cos \left(r_{2}\right)\left|r_{3} g_{*}-x_{i}^{t}\right|, & r_{4} \leq 0.5 .
\end{array}
$$

Here $r_{1}, r_{2}, r_{3}$, and $r_{4}$ are parameters that are fitted to have a promising search region. Parameter $r_{1}$ dictates the next position regions (or movement direction) which could be either in the space between the solution and destination or outside it, set as $r_{1}=(T-t) a / T$, where $t$ is the current iteration, $T$ is the maximum number of iteration, and $a$ is a constant. The parameter $r_{2}=a * \pi *$ rand defines how far the movement should be towards or outwards the destination. The parameter $r_{3}=a *$ rand gives random weights that are being introduced for the purpose of bringing the solution either towards the best or away from the best. If the value of $r_{3}$ is greater than 1, the current solution will be away from the best solution, whereas if $r_{3}$ has a value less than 1 , the best solution will be much nearer to the current solution. Finally, the parameter $r_{4}$ is a random number between $[0,1]$ and it is used to switch between the Cosine and Sine components.

Using equation (8) to replace the switch probability in Rule 4 of Flower Pollination Algorithm (FPA), and equation (9) and (10) in place of equation (5), we have an improved version of the algorithm called Pollination Intelligence Algorithm (PIA) which will be used together with Flower Pollinated Algorithm (FPA) and LINGO optimization modeling tool to solve the Agricultural Land Model in equations (1)-(4) (Algorithm 2).

To implement the model, a case study of a farmer in Ife, Osun State, Nigeria, was used, data showing cost of production were collected from the farmer's farm diary and record book, and it is presented in Tables 1-3. The farmer is known to plant crops that can be grown in the region. Yield data for the selected crops were collected from http://www.nigeria.opendataforafrica.org, and the average yields ( $\mathrm{kg} / \mathrm{acre}$ ) of the selected crops (maize, cassava, 


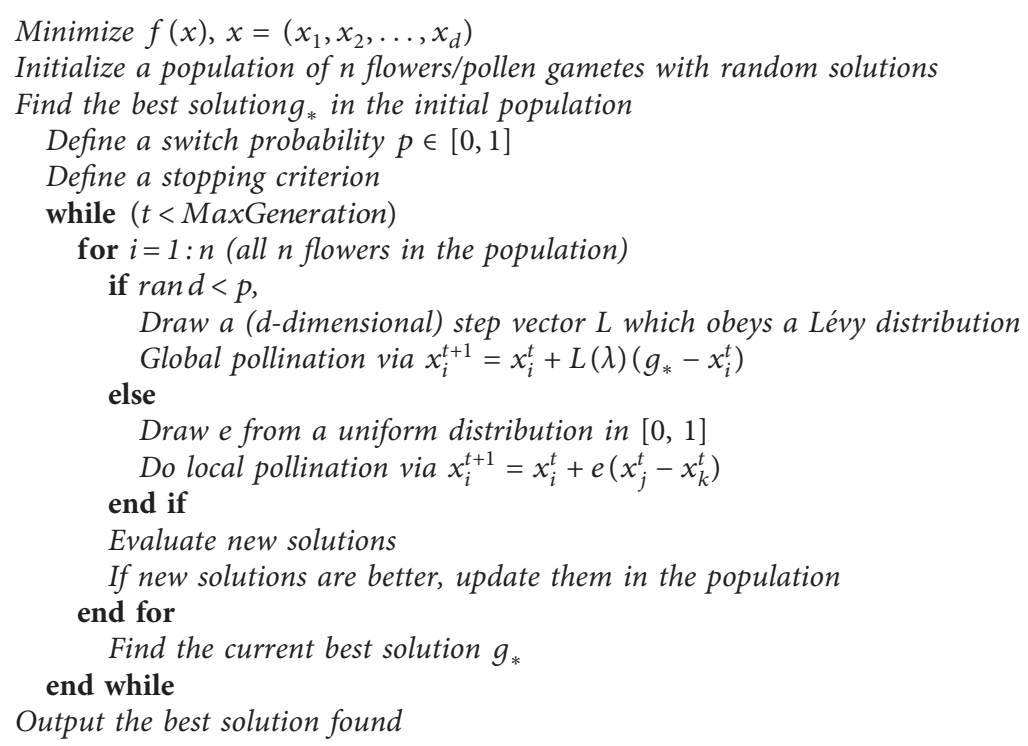

Algorithm 1: Flower Pollinated Algorithm by Yang [15].

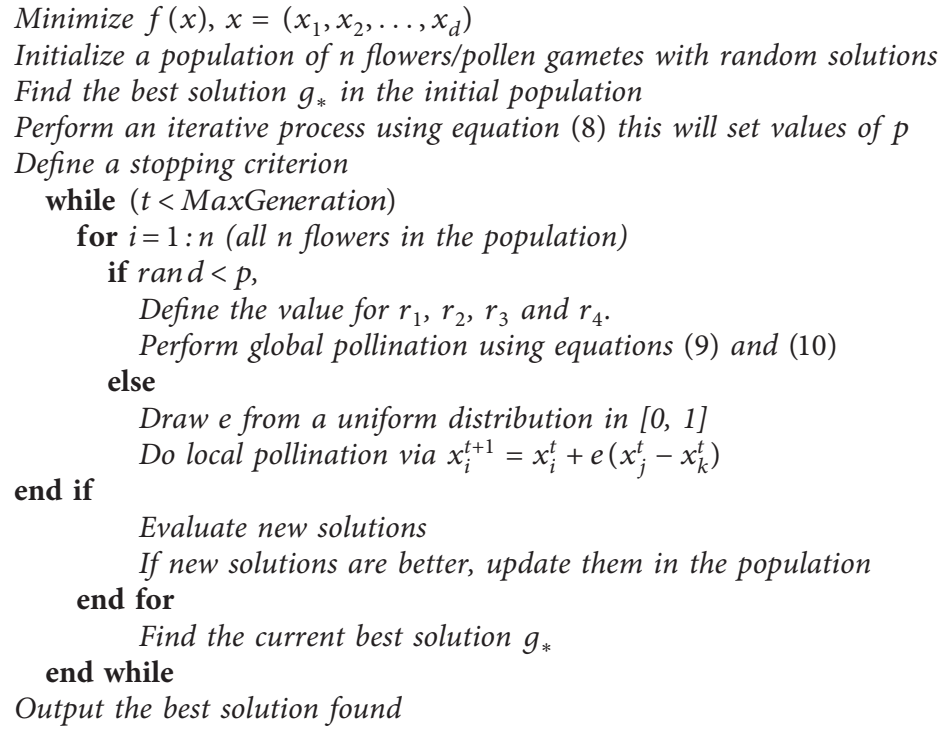

Algorithm 2: Pollination Intelligence Algorithm.

Table 1: Dominion farm, Ife, Osun State, Nigeria (cost of planting maize per acre).

\begin{tabular}{lc}
\hline Item & Cost (Naira) \\
\hline Clearing of land & $13,000.00$ \\
Maize seedlings & $2,500.00$ \\
Insecticide & $3,000.00$ \\
Workmanship & $5,000.00$ \\
Total cost of planting & $\mathbf{2 3 , 5 0 0 . 0 0}$ \\
Total income & $85,400.00$ \\
Profit & $\mathbf{6 1 , 9 0 0 . 0 0}$ \\
\hline
\end{tabular}

and yam) in Nigeria are 233.695, 1358.81, and 1282.305, respectively, and the market price (per $\mathrm{kg}$ ) of the selected crops is 160, 120 and 200 which was selected by visiting IleIfe Market in Nigeria. The farmer's records showing cost of planting for the selected crops are presented in Tables 1-3.

\section{Results and Discussion}

We used LINGO optimizer, Flower Pollinated Algorithm (FPA), and the new method Pollination Intelligence Algorithm (PIA) to solve the model, and the obtained results 
TABle 2: Dominion farm, Ife, Osun State, Nigeria (cost of planting cassava per acre).

\begin{tabular}{lc}
\hline Item & Cost (Naira) \\
\hline Clearing of land & $13,000.00$ \\
Making of ridges & $10,000.00$ \\
Cassava stem & $4,000.00$ \\
Workmanship & $6,500.00$ \\
Herbicide & $2,500.00$ \\
Total cost of planting & $\mathbf{3 6 , 0 0 0 . 0 0}$ \\
Total income & $146,000.00$ \\
Profit & $\mathbf{1 1 0 , 0 0 0 . 0 0}$ \\
\hline
\end{tabular}

Table 3: Dominion farm, Ife, Osun State, Nigeria (cost of planting yam per acre).

\begin{tabular}{lc}
\hline Item & Cost (Naira) \\
\hline Clearing of land & $13,000.00$ \\
Making of ridges & $10,000.00$ \\
Yam seedlings & $8,000.00$ \\
Workmanship & $10,000.00$ \\
Herbicide & $3,000.00$ \\
Pesticide & $3,000.00$ \\
Total cost of planting & $\mathbf{4 7 , 0 0 0 . 0 0}$ \\
Total income & $203,000.00$ \\
Profit & $\mathbf{1 5 6 , 0 0 0 . 0 0}$
\end{tabular}

were compared with farmer's intuition of allocating crops and profit earned from maize, cassava, and yam plantation, respectively, using 3 acres as used by the farmer. Results from Table 4 show the optimal allocation of crops to agricultural land for cultivation and the profit obtained by solving the model equation using the solvers and the methods. Meanwhile, results obtained in Table 4 show that the farmland has been wasted following the farmers' intuition process. The amount of land allocated to maize and cassava was out of hand to not guarantee the desired profit. The land would have best been maximized if yam has been given a priority following the nature of its yield and market price demand. Figures 1 and 2 show the allocation of crops and profit obtained from the model using the available number of lands used by the farmer for different crops selected based on his intuition and the optimal allocation obtained from the model using LINGO, Flower Pollinated Algorithm (FPA), and Pollination Intelligence Algorithm (PIA). Figures 1 and 2 establish that metaheuristic method of optimization can best be used in solving land allocation problems as crops were optimally allocated to the available land space for profit generation as against the result obtained from LINGO software which has the characteristics of the traditional method of optimization. The further result from these figures shows that optimal profit and allocation was achieved using the newly developed Pollination Intelligence Algorithm and solution obtained was very close to that of Flower Pollination Algorithm with a profit of 751,970 Naira and 751,300 Naira, respectively. Pollination Intelligence Algorithm (PIA) from this result displays superiority over the other methods used in solving the model.
TABLE 4: Result of crop acreage and profit obtained from the model.

\begin{tabular}{lcccc}
\hline Land (acres) & Farmer & Lingo & FPA & PIA \\
& 3 & 3 & 3 & 3 \\
\hline Maize (acre) & 1 & 0.1460594 & 0.012399 & 0.006299 \\
Cassava (acre) & 1 & 1.368810 & 0.010150 & 0.016452 \\
Yam (acre) & 1 & 1.485131 & 2.977450 & 2.977242 \\
Profit (Naira) & $327,900.00$ & $561,452.40$ & $751,300.00$ & $751,970.00$ \\
\hline
\end{tabular}

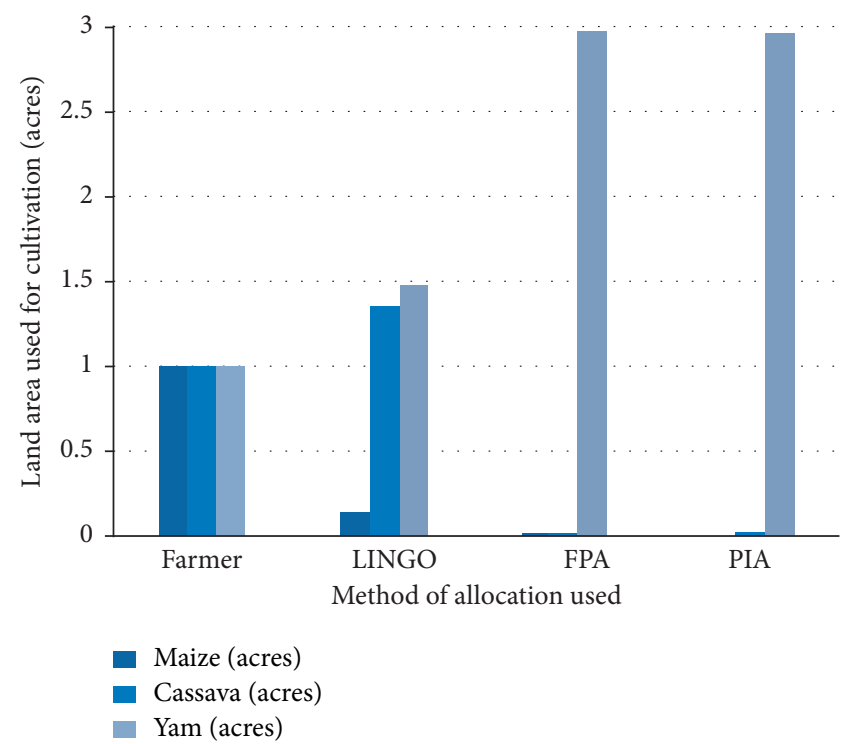

Figure 1: Chart showing allocation of crops.

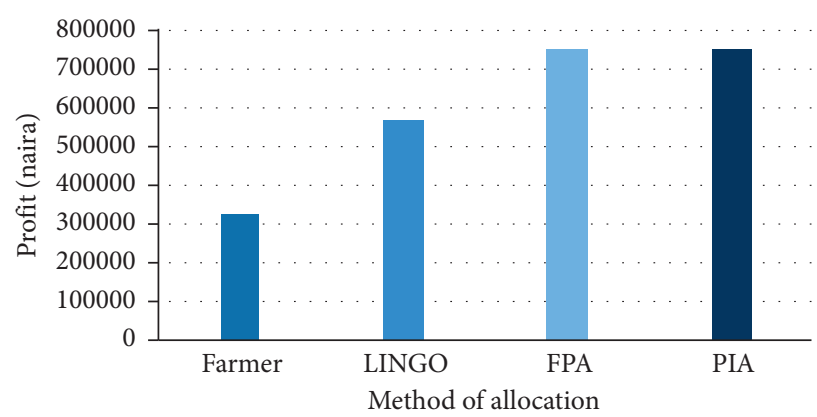

Figure 2: Chart showing profits obtained.

\section{Conclusion}

An agricultural model for allocating crops is presented in this work. The developed model was solved using LINGO, Flower Pollination Algorithm by Yang [15], and a newly designed method called Pollination Intelligence Algorithm. Results from Table 4 gave an insight into the farmer's decision in assigning crops of interest to his portion of land for cultivation. The Improved Flower Pollinated Algorithm called Pollination Intelligence Algorithm had a result relatively close to the solution obtained from Flower Pollinated Algorithm by Yang [15] and produces more profit from the assigned crop acreage. It is worthwhile to note that more iterative schemes can be developed to extend Flower 
Pollinated Algorithm and a more distributed function having the same properties with Sine Cosine operator and Lévy flight can be used to mimic the movement of pollinating agents. Furthermore, it can be deduced from the result that metaheuristic method of optimization has the ability of solving crop allocation problems, and instead of farmers using the intuition process in farm management, necessary factors must be put in place before embarking on farming activities.

\section{Abbreviations}

I: $\quad$ Number of selectable crops

$P_{i}$ : $\quad$ Price of crops $i$

$Y_{i}$ : Yield of crop $i$

$Y_{i}^{p}$ : Projected yield of crop $i$

$\mathrm{C}_{i}$ : Variable cost of planting crop $i$

$F_{c}^{L}$ : Fixed cost of cultivating the whole land

$\mathrm{A}_{i}$ : Area of land assigned to crop $i$

L: Total amount of land available for cultivation.

\section{Data Availability}

The data used to support the findings of the study are included within the article.

\section{Conflicts of Interest}

This research work is self-sponsored by the authors with reference materials well cited accordingly. There are no conflicts of interest regarding the publication of this paper.

\section{References}

[1] H. Aimin, "Uncertainty, risk aversion and risk management in agriculture," Agriculture and Agricultural Science Procedia, vol. 1, pp. 152-156, 2010.

[2] J. L. Dillon, “The definition of farm management," Journal of Agricultural Economics, vol. 31, no. 2, pp. 257-258, 1980.

[3] R. Krishna, "The Optimality of land allocation: a case study of Punjab," Indian Journal of Agricultural Economics, vol. 18, no. 1, pp. 63-73, 1963.

[4] B. Keith, "Practical application of linear/integer programing in agriculture," Journal of Operational Research Society, vol. 36, no. 2, pp. 99-107, 1985.

[5] J. C. Campbell, J. Radke, J. T. Gless, and R. M. Wirtshafter, "An application of linear programming and geographic information systems: cropland allocation in antigua," Environment and Planning A: Economy and Space, vol. 24, no. 4, pp. 535-549, 1992.

[6] I. Sante and R. Crecente, "A review of rural land use planning model," Environmental and Planning B: Planning and Designs, vol. 33, no. 1, pp. 165-183, 2006.

[7] M. O. Wankhade and H. S. Lunge, "Allocation of agricultural land to the major crops of saline track by linear programming approach: a case study," International Journal of Scientific and Technology Research, vol. 1, no. 9, pp. 21-25, 2012.

[8] M. A. Ahmed, I. A. Ahmed, and S. A. Fawzi, "Optimization of the cropping pattern in Saudi Arabia using a mathematical programing sector model," Agricultural Economics (Zemědělská ekonomika), vol. 58, no. 2, pp. 56-60, 2012.
[9] J. H. Holland, Adaptation in Natural and Artificial Systems, University of Michigan Press, Ann Arbor, MI, USA, 1975.

[10] S. Kirkpatrick, J. Gelatt, and M. Vecchi, "Optimization by simulated annealing," Journal of Science, vol. 220, no. 4798, pp. 671-680, 1983.

[11] R. Storn and K. Price, "Differential evolution, a simple and efficient heuristic for global optimization over continuous spaces," Journal of Global Optimization, vol. 11, no. 4, pp. 341-359, 1997.

[12] X. S. Yang, Nature Inspired Metaheuristic Algorithm, Luniver press, Cambridge, UK, 2008.

[13] Z. Bayraktar, M. Komurcu, and D. H. Werner, "Wind driven optimization: a novel nature-inspired optimization algorithm and its application to electromagnetic," in Proceedings of the Antennas and Propagation Society International Symposium (APSURSI), pp. 1-4, IEEE, Toronto, Canada, July 2010.

[14] Y. Shi, "An optimization algorithm based on brainstorming process," International Journal of Swarm Intelligence Research, vol. 2, no. 4, pp. 35-62, 2011.

[15] X.-S. Yang, "Flower pollination algorithm for global optimization," Unconventional Computation and Natural Computation, vol. 7445, pp. 240-249, 2012.

[16] I. Hassan, M. A. Raza, and R. Ilahi, "Use of linear programing model to determine the optimum cropping pattern, production and income level: a case study from Dera Ghazi Khan division," Journal of Agriculture and Social Sciences, vol. 1, pp. 1813-2235, 2005.

[17] L. Tanko, C. E. Onyenweakwu, and A. C. Nwosu, "Optimum crop combination under limited resources condition: a microlevel study in Yauri, Kebbi state Nigeria," Nigeria Agricultural Journa, vol. 37, no. 1, pp. 1-10, 2006.

[18] P. Nedunchezhian and M. Thirunarukkarasu, "Optimizing farm plans in different farming system," Agricultural Economics Research Review, vol. 20, pp. 147-156, 2007.

[19] B. Manos, J. Papathanasiou, T. Bournaris, and K. Voudouris, "A multicriteria model for planning agricultural regions within a context of groundwater rational management," Journal of Environmental Management, vol. 91, no. 7, pp. 1593-1600, 2010.

[20] K. C. Igwe, C. E. Onyeweakwu, and S. Nwosu, "Application of linear programming to semi-commercial arable and fishery enterprises in Abia state, Nigeria," International Journal of Economics and Management Sciences, vol. 1, no. 1, pp. 75-81, 2011.

[21] O. M. Bamiro, M. Afolabi, and F. Daramola, "Enterprise combinations in cassava based food crop farming system in Nigeria: evidence from Ogun state," Greener Journal of Agricultural Sciences, vol. 2, no. 1, pp. 13-20, 2012.

[22] F. Majeke, J. Majeke, N. Chabuka et al., "A farm resource allocation problem. A case study of model A2 resettled farmers in bindura, Zimbabwe," International Journal of Economics and Management Science, vol. 2, no. 7, pp. 1-4, 2013.

[23] I. Badarudin, A. M. Sultan, M. N. Sulaiman, and M. F. Mohammed, "Metaheuristic approach for optimizing agricultural land," in Proceedings of the 2009 2nd Conference on Data Mining and Optimization, IEEE, Kajand, Malaysia, Ocotober 2009.

[24] S. Mirjalili, "SCA: a sine cosine algorithm for solving optimization problems," Knowledge-Based System, vol. 96, pp. 120-133, 2016. 